\title{
Probabilistic Approaches for Estimating Surface Roughness in Multi-pass Horizontal Grinding Operations
}

\author{
H.G.Choi, M.K.Yeo, J.Y.Kim
}

\begin{abstract}
Sung Kyun Kwan University, Department of Indusrtrial Engineering Suwon Kyong Ki Do 440-746 S.Korea, Phone number:(nation code)-331-290-7616, Fax number:(nation code)-331-290-7610, E-mail address:hgchoi@yurim.skku.ac.kr.
\end{abstract}

\begin{abstract}
The grinding operation is one of the most popular finishing operations, which requires high costs and large machining times to achieve a given surface roughness. Although the operation has been used to produce better surfaces after several interim operations, a few theoretical and practical research about its operational characteristics has been presented. The surface roughness resulted by an abrasive wheel is the typical characteristic. This study presents mathematical models to estimate probabilistically surface roughness in multi-pass horizontal surface grinding operations from the initial surface roughness distributions of a workpiece, the distributions of the wheel radius, and the distributions of distances between active grains. As a result, we can show either the probability satisfying a given surface roughness or the range of surface roughness satisfying a given probability when grinding conditions are fixed. Also, we can establish the relationship between grinding conditions satisfying the range of surface roughness under a given probability.
\end{abstract}

Keywords

Probabilistic Estimation, Surface Roughness, Multi-pass Grinding Operations

\section{Introduction}

The surface roughness in grinding operations is determined by a combined effects of irregular factors of a grinding wheel such as rotational speed, feed rate, 
diameter, active grain size, and distance between active grains. Vibration, thermal effect, and friction between the wheel and a workpiece are another factors to affect the roughness. A lot of existing studies have reported the most recommendable roughness values to be obtained experimentally or empirically. Recently, however, the need of mathematical models for estimating the surface roughness has been exposed to reduce inaccurate estimation or ambiguous decisions in grinding areas.[1][4] Several features of grinding operations have been represented mathematically or statistically. Law and et.al[3] presented that the distance between neighboring active grains follows an exponential distribution and a sum of the wheel radius and the active grain size follows a $\mathrm{Z}$ distribution. Malkin[4] presented that the irregularities on a workpiece surface come from the wheel characteristics such as grain sizes, wheel radius, wheel material, etc. Also, he suggested uniform distributions for both the distance between neighboring active grains and the active grain sizes because the grain extraction and bonding methods have been evolved to make more uniform wheels.

In this study, a probabilistic modeling approach is used for estimating accurately surface roughness under mathematical characteristics of a wheel. A horizontal grinding machine is assumed to perform grinding operations. A worktable with a loaded workpiece moves back and forth to obtain a specified surface roughness while a wheel continuously rotates. It is hard to achieve a given roughness by one pass of the worktable, and so multiple number of passes are usually required. The following assumptions are adopted to estimate surface roughness in horizontal grinding operation:

1. The number of passes will not affect to the wheel characteristics.

2. The active grain size is smaller than the initial surface roughness of a workpiece in multi-pass cases.

3. The cutting fluids and wheel dressing are not applied.

4. The surface roughness is not affected by irregular factors such as vibration, thermal effect, friction, etc.

Assumptions 1., 2., and 4. describe that no factors related to the wheel wear are considered. The second assumption means that the initial surface roughness of a workpiece is ground repeatedly to obtain a desired roughness.

\section{Ideal Surface Roughness in Horizontal Grind- ing Operations}

In this study, the ideal surface roughness equation presented by Malkin[4] has been used for deriving a probability density function of surface roughness. The 
ideal surface roughness[4] depicted in Figure 1 is determined by Equation (1). Every pass of the grinding wheel over a workpiece creates a new roughness represented by Equation (1).

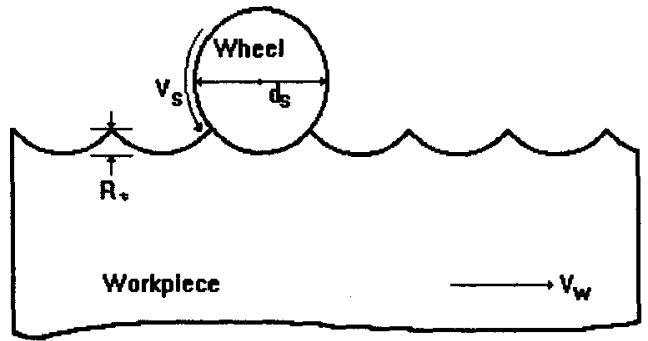

Figure 1: Ideal surface roughness[4].

$R_{t}=\frac{1}{4}\left[\frac{V_{w} X}{\sqrt{2} V_{o} Y^{1 / 2}}\right]^{2}=\frac{V_{w}^{2}}{8 V_{0}^{2}} Z=\alpha Z \quad\left(\alpha=\frac{V_{w}^{2}}{8 V_{s}^{2}}\right)$

Where $R_{t}=$ ideal surface roughness(peak-to-valley surface roughness), $V_{s}=$ rotational speed of a spindle or a grinding wheel, $V_{w}=$ work table speed, $X=$ distance between neighboring active grains, $Y=\mathrm{ds} / 2, Z=X^{2} / Y, d_{s}=$ wheel diameter.

$R_{t}$ is a function of $Z$ and is a random variable. When a statistical distribution is defined for the distance between the active grains or for the wheel radius, a probability density function of surface roughness $f_{U}(u)$ is defined for multi-pass cases.

\section{Probabilistic Models}

When a workpiece surface is ground several times to obtain a given roughness, the $(\mathrm{n}-1)$ th roughness is affect to the nth roughness where $\mathrm{n}$ is the number of travels required for a worktable. In this study, the initial surface roughness $(T)$ is set as a uniform distribution. The highest roughness and the lowest roughness of the initial surface are $h$ and $g$, respectively. Then, the roughness is reduced in every pass as much as the constant amount, $R_{t}$.

$$
f_{T}(t)=\frac{1}{h-g} I_{(g, h)}(t), \quad R_{t}^{[n]}: \text { surface roughness after the } n \text {th pass }
$$

where $I_{(g, h)}(t)$ is an indication function which is defined by either 1 in the range of $g \leq t \leq h$ or 0 in other ranges. 


$$
\begin{gathered}
T-R_{t}=R_{t}^{[2]} \\
\mathrm{R}_{t}^{[1]}-R_{t}=R_{t}^{[2]} \\
\mathrm{R}_{t}^{[2]}-R_{t}=R_{t}^{[3]} \\
\cdot \\
\cdot \\
R_{t}^{[n-1]}-R_{t}=R_{t}^{[n]} .
\end{gathered}
$$

Then, these equations are combined together and simplified as follows:

$$
\begin{aligned}
T-2 R_{t} & =R_{t}^{[2]} \quad\left(T-R_{t}=R_{t}^{[1]}\right) . \\
T-3 R_{t} & =R_{t}^{[3]} . \\
\cdot & \\
\cdot & \\
T-n R_{t} & =R_{t}^{[n]} . \\
T-n R_{t} & =T-\beta Z=U \quad\left(\beta=\frac{n V_{v}^{2}}{8 V_{s}^{2}}, U=R_{t}^{[n]}\right) .
\end{aligned}
$$

Both $T$ and $U$ are random variables. Then, the p.d.f of surface roughness is defined by $f_{U}(u)$ for multi-pass cases.

\subsection{Uniform distance and uniform radius}

Both the distance between neighboring active grains, $X$ and the wheel radius, $Y$ are defined as uniform distributions,

$$
f_{X}(x)=\frac{1}{b-a} I_{(a, b)}(x), f_{Y}(y)=\frac{1}{d-c} I_{(c, d)}(y), K_{2}=3(b-a)(d-c)(h-g)
$$

where $I_{(a, b)}(x)$ is an indication function that is defined by either 1 in the range of $a \leq X \leq b$ or 0 in other ranges. $I_{(c, d)}(y)$ is defined similarly. Then, two separated p.d.f must be defined as follows:

$$
\begin{aligned}
\text { - } & \text { CASE I }\left(\frac{b^{2}}{d}<\frac{a^{2}}{c}\right) \\
& f_{U}(u)=\frac{1}{K_{2}}\left\{-3 b c+2 c \sqrt{c} \sqrt{\frac{g-u}{\beta}}+\frac{\beta b^{3}}{g-u}\right\} I_{\left(g-\frac{\beta b^{2}}{c}, g-\frac{\beta a^{2}}{c}\right)}(U) \\
+ & \frac{1}{K_{2}}\left\{3 a c-3 b c+\frac{\beta b^{3}}{g-u}-\frac{\beta a^{3}}{g-u}\right\} I_{\left(g-\frac{\beta a^{2}}{c}, g-\frac{\beta b^{2}}{d}\right)}(U) \\
+ & \frac{1}{K_{2}}\left\{3 a c+3 b d-3 b c-2 d \sqrt{d} \sqrt{\frac{g-u}{\beta}}-\frac{\beta a^{3}}{g-u}\right\} I_{\left(g-\frac{\beta b^{2}}{d}, g-\frac{\beta a^{2}}{d}\right)}(U) \\
+ & \frac{1}{h-g} I_{\left(g-\frac{\beta a^{2}}{d}, h-\frac{\beta b^{2}}{c}\right)}(U) \\
+ & \frac{1}{K_{2}}\left\{3 a c+3 b d-3 a d-2 c \sqrt{c} \sqrt{\frac{h-u}{\beta}}-\frac{\beta b^{3}}{h-u}\right\} I_{\left(h-\frac{\beta b^{2}}{c}, h-\frac{\beta a^{2}}{c}\right)}(U) \\
+ & \frac{1}{K_{2}}\left\{3 b d-3 a d+\frac{\beta a^{3}}{h-u}-\frac{\beta b^{3}}{h-u}\right\} I_{\left(h-\frac{\beta a^{2}}{c}, h-\frac{\beta b^{2}}{d}\right)}(U)
\end{aligned}
$$




$$
\begin{aligned}
& +\frac{1}{K_{2}}\left\{-3 a d+2 d \sqrt{d} \sqrt{\frac{h-u}{\beta}}+\frac{\beta a^{3}}{h-u}\right\} I_{\left(h-\frac{\beta b^{2}}{d}, h-\frac{\beta a^{2}}{d}\right)}(U) \\
& \text { - CASE II }\left(\frac{a^{2}}{c}<\frac{b^{2}}{d}\right) \\
& f_{U}(u)=\frac{1}{K_{2}}\left\{-3 b c+2 c \sqrt{c} \sqrt{\frac{g-u}{\beta}}+\frac{\beta b^{3}}{g-u}\right\} I_{\left(g-\frac{\beta b^{2}}{c}, g-\frac{\beta b^{2}}{d}\right)}(U) \\
& +\frac{1}{K_{2}}\left\{3 b d-3 b c+2(c \sqrt{c}-d \sqrt{d}) \sqrt{\frac{g-u}{\beta}}\right\} I_{\left(g-\frac{\beta b^{2}}{d}, g-\frac{\beta a^{2}}{c}\right)}(U) \\
& +\frac{1}{K_{2}}\left\{3 a c+3 b d-3 b c-2 d \sqrt{d} \sqrt{\frac{g-u}{\beta}}-\frac{\beta a^{3}}{g-u}\right\} I_{\left(g-\frac{\beta a^{2}}{c}, g-\frac{\beta a^{2}}{d}\right)}(U) \\
& +\frac{1}{h-g} I_{\left(g-\frac{\beta a^{2}}{d}, h-\frac{\beta b^{2}}{c}\right)}(U) \\
& +\frac{1}{K_{2}}\left\{3 a c+3 b d-3 a d-2 c \sqrt{c} \sqrt{\frac{h-u}{\beta}}-\frac{\beta b^{3}}{h-u}\right\} I_{\left(h-\frac{\beta b^{2}}{c}, h-\frac{\beta b^{2}}{d}\right)}(U) \\
& +\frac{1}{K_{2}}\left\{3 a c-3 a d+2(d \sqrt{d}-c \sqrt{c}) \sqrt{\frac{h-u}{\beta}}\right\} I_{\left(h-\frac{\beta b^{2}}{d}, h-\frac{\beta a^{2}}{c}\right)}(U) \\
& +\frac{1}{K_{2}}\left\{-3 a d+2 d \sqrt{d} \sqrt{\frac{h-u}{\beta}}+\frac{\beta a^{3}}{h-u}\right\} I_{\left(h-\frac{\beta a^{2}}{c}, h-\frac{\beta a^{2}}{d}\right)}(U)
\end{aligned}
$$

\subsection{Exponential distance and uniform radius}

When the p.d.f of the distance between neighboring active grains and the p.d.f of the wheel radius are given respectively as follows:

$$
f_{X}(x)=\lambda e^{-\lambda x}, f_{Y}(y)=\frac{1}{d-c} I_{(c, d)}(y), K_{3}=\left(e^{-\lambda a}-e^{-\lambda b}\right)(d-c)(h-g)
$$

the p.d.f of $f_{U}(u)$ for Case I and Case II are obtained.

- CASE I $\left(\frac{b^{2}}{d}<\frac{a^{2}}{c}\right)$

$$
\begin{aligned}
& f_{u}(u)=\left\{\frac{\lambda b e^{-\lambda b}\left(20 b^{2}+5 b^{9} \lambda+b^{4} \lambda^{2}\right)}{60 K_{3}} \frac{\beta}{g-u}+\frac{2 \lambda c \sqrt{c}}{3 K_{3}} \sqrt{\frac{g-u}{\beta}} e^{-\lambda \sqrt{c} \sqrt{\frac{g-u}{\beta}}}\right. \\
& +\frac{5 \lambda a^{2} c^{2}}{12 K_{3}} \frac{g-u}{\beta} e^{-\lambda \sqrt{c} \sqrt{\frac{g-u}{\beta}}}+\frac{3 \lambda a^{3} c^{2} \sqrt{c}}{20 K_{3}} \sqrt{\frac{g-u}{\beta}} \frac{g-u}{\beta} e^{-\lambda \sqrt{c} \sqrt{\frac{g-u}{\beta}}} \\
& +\frac{7 \lambda a^{4} c^{2}}{720 K_{3}}\left(\frac{g-u}{\beta}\right)^{2} e^{-\lambda \sqrt{c} \sqrt{\frac{g-u}{\beta}}}+\frac{\lambda a^{5} c^{3} \sqrt{c}}{1800 K_{3}} \sqrt{\frac{g-u}{\beta}}\left(\frac{g-u}{\beta}\right)^{2} e^{-\lambda \sqrt{c} \sqrt{\frac{g-u}{\beta}}} \\
& \left.-\frac{\lambda b c e^{-\lambda b}}{K_{3}}-\frac{\lambda^{2} b^{2} c e^{-\lambda b}}{2 K_{3}}-\frac{\lambda^{3} b^{3} c e^{-\lambda b}}{6 K_{3}}-\frac{7 \lambda^{4} b^{4} c e^{-\lambda b}}{720 K_{3}}-\frac{\lambda^{5} b^{5} c e^{-\lambda b}}{1800 K_{3}}\right\} I_{\left(g-\frac{\beta b^{2}}{c}, g-\frac{\beta a^{2}}{c}\right)}(u) \\
& +\left\{\frac{\lambda b e^{-\lambda b}\left(20 b^{2}+5 b^{3} \lambda+b^{4} \lambda^{2}\right)-\lambda a e^{-\lambda a}\left(20 a^{2}+5 a^{3} \lambda+a^{4} \lambda^{2}\right)}{60 K_{3}} \frac{\beta}{g-u}\right. \\
& -\frac{\lambda b c e^{-\lambda b}}{K_{3}}-\frac{\lambda^{2} b^{2} c e^{-\lambda b}}{2 K_{3}}-\frac{\lambda^{3} b^{3} c e^{-\lambda b}}{6 K_{3}}-\frac{7 \lambda^{4} b^{4} c e^{-\lambda b}}{720 K_{3}}-\frac{\lambda^{5} b^{5} c e^{-\lambda b}}{1800 K_{3}} \\
& \left.+\frac{\lambda a c e^{-\lambda a}}{K_{3}}+\frac{\lambda^{2} a^{2} c e^{-\lambda a}}{2 K_{3}}+\frac{\lambda^{3} a^{3} c e^{-\lambda a}}{6 K_{3}}+\frac{7 \lambda^{4} a^{4} c e^{-\lambda a}}{720 K_{3}}+\frac{\lambda^{5} a^{5} c e^{-\lambda a}}{1800 K_{3}}\right\} I_{\left(g-\frac{\beta a^{2}}{c}, g-\frac{\beta b^{2}}{d}\right)}(u) \\
& +\left\{-\frac{\lambda a e^{-\lambda a}\left(20 a^{2}+5 a^{3} \lambda+a^{4} \lambda^{2}\right)}{60 K_{3}} \frac{\beta}{g-u}-\frac{2 \lambda d \sqrt{d}}{3 K_{3}} \sqrt{\frac{g-u}{\beta}} e^{-\lambda \sqrt{d} \sqrt{\frac{g-u}{\beta}}}\right. \\
& -\frac{5 \lambda^{2} d^{2}}{12 K_{3}} \frac{q-u}{\beta} e^{-\lambda \sqrt{d} \sqrt{\frac{2-u}{\beta}}}-\frac{3 \lambda^{3} d^{2} \sqrt{d}}{20 K_{3}} \sqrt{\frac{q-u}{\beta} \frac{q-u}{\beta}} e^{-\lambda \sqrt{d} \sqrt{\frac{g-u}{\beta}}}
\end{aligned}
$$




$$
\begin{aligned}
& -\frac{7 \lambda^{4} d^{3}}{720 K_{3}}\left(\frac{g-u}{\beta}\right)^{2} e^{-\lambda \sqrt{d} \sqrt{\frac{g-u}{\beta}}}-\frac{\lambda^{5} d^{3} \sqrt{d}}{1800 K_{3}} \sqrt{\frac{q-u}{\beta}}\left(\frac{q-u}{\beta}\right)^{2} e^{-\lambda \sqrt{d} \sqrt{\frac{g-u}{\beta}}} \\
& -\frac{\lambda b c e^{-\lambda b}}{K_{3}}-\frac{\lambda^{2} b^{2} c e^{-\lambda b}}{2 K_{3}}-\frac{\lambda^{3} b^{3} c e^{-\lambda b}}{6 K_{3}}-\frac{7 \lambda^{4} b^{4} c e^{-\lambda b}}{720 K_{3}}-\frac{\lambda^{5} b^{5} c e^{-\lambda b}}{1800 K_{3}} \\
& +\frac{\lambda a c e^{-\lambda a}}{K_{3}}+\frac{\lambda^{2} a^{2} c e^{-\lambda a}}{2 K_{3}}+\frac{\lambda^{3} a^{3} c e^{-\lambda a}}{6 K_{3}}+\frac{7 \lambda^{4} a^{4} c e^{-\lambda a}}{720 K_{3}}+\frac{\lambda^{5} a^{5} c e^{-\lambda a}}{1800 K_{3}} \\
& \left.+\frac{\lambda b d e^{-\lambda b}}{K_{3}}+\frac{\lambda^{2} b^{2} d e^{-\lambda b}}{2 K_{3}}+\frac{\lambda^{3} b^{3} d e^{-\lambda b}}{6 K_{3}}+\frac{7 \lambda^{4} b^{4} d e^{-\lambda b}}{720 K_{3}}+\frac{\lambda^{5} b^{5} d e^{-\lambda b}}{1800 K_{3}}\right\} I_{\left(g-\frac{\beta b^{2}}{d}, g-\frac{\beta a^{2}}{d}\right)}(u) \\
& +\left\{\frac { 1 } { ( e ^ { - \lambda a } - e ^ { - \lambda b } ) ( h - g ) } \left(\lambda b e^{-\lambda b}-\lambda a e^{-\lambda a}+\frac{\lambda^{2} b^{2} e^{-\lambda b}}{2}-\frac{\lambda^{2} a^{2} e^{-\lambda a}}{2}+\frac{\lambda^{3} b^{3} e^{-\lambda b}}{6}-\frac{\lambda^{3} a^{3} e^{-\lambda a}}{6}\right.\right. \\
& \left.\left.+\frac{\lambda^{4} b^{4} e^{-\lambda b}}{720}-\frac{\lambda^{4} a^{4} e^{-\lambda a}}{720}+\frac{\lambda^{5} b^{5} e^{-\lambda b}}{1800}-\frac{\lambda^{5} a^{5} e^{-\lambda a}}{1800}\right)\right\} I_{\left(g-\frac{\beta a^{2}}{d}, h-\frac{\beta b^{2}}{c}\right)}(u) \\
& +\left\{-\frac{\lambda b e^{-\lambda b}\left(20 b^{2}+5 b^{3} \lambda+b^{4} \lambda^{2}\right)}{60 K_{3}} \frac{\beta}{h-u}-\frac{2 \lambda c \sqrt{c}}{3 K_{3}} \sqrt{\frac{h-u}{\beta}} e^{-\lambda \sqrt{c} \sqrt{\frac{h-u}{\beta}}}\right. \\
& -\frac{5 a^{2} c^{2}}{12 K_{3}} \frac{h-u}{\beta} e^{-\lambda \sqrt{c} \sqrt{\frac{h-u}{\beta}}}-\frac{3 \lambda^{3} d^{2} \sqrt{c}}{20 K_{3}} \sqrt{\frac{h-u}{\beta}} \frac{h-u}{\beta} e^{-\lambda \sqrt{c} \sqrt{\frac{h-u}{\beta}}} \\
& -\frac{7 a^{4} c^{3}}{720 K_{3}}\left(\frac{h-u}{\beta}\right)^{2} e^{-\lambda \sqrt{c} \sqrt{\frac{h-u}{\beta}}}-\frac{\lambda^{5} c^{3} \sqrt{c}}{1800 K_{3}} \sqrt{\frac{h-u}{\beta}}\left(\frac{h-u}{\beta}\right)^{2} e^{-\lambda \sqrt{c} \sqrt{\frac{h-u}{\beta}}} \\
& -\frac{\lambda a d e^{-\lambda a}}{K_{3}}-\frac{\lambda^{2} a^{2} d e^{-\lambda a}}{2 K_{3}}-\frac{\lambda^{3} a^{3} d e^{-\lambda a}}{6 K_{3}}-\frac{7 \lambda^{4} a^{4} d e^{-\lambda a}}{720 K_{3}}-\frac{\lambda^{5} a^{5} d e^{-\lambda a}}{1800 K_{3}} \\
& +\frac{\lambda a c e^{-\lambda a}}{K_{3}}+\frac{\lambda^{2} a^{2} c e^{-\lambda a}}{2 K_{3}}+\frac{\lambda^{3} a^{3} c e^{-\lambda a}}{6 K_{3}}+\frac{7 \lambda^{4} a^{4} c e^{-\lambda a}}{720 K_{3}}+\frac{\lambda^{5} a^{5} c e^{-\lambda a}}{1800 K_{3}} \\
& \left.+\frac{\lambda b d e^{-\lambda b}}{K_{3}}+\frac{\lambda^{2} b^{2} d e^{-\lambda b}}{2 K_{3}}+\frac{\lambda^{3} b^{3} d e^{-\lambda b}}{6 K_{3}}+\frac{7 \lambda^{4} b^{4} d e^{-\lambda b}}{720 K_{3}}+\frac{\lambda^{5} b^{5} d e^{-\lambda b}}{1800 K_{3}}\right\} I_{\left(h-\frac{\beta b^{2}}{c}, h-\frac{\beta a^{2}}{c}\right)}(u) \\
& +\left\{\frac{\lambda a e^{-\lambda a}\left(20 a^{2}+5 a^{3} \lambda+a^{4} \lambda^{2}\right)-\lambda b e^{-\lambda b}\left(20 b^{2}+5 b^{3} \lambda+b^{4} \lambda^{2}\right)}{60 K_{3}} \frac{\beta}{h-u}\right. \\
& -\frac{\lambda a d e^{-\lambda a}}{K_{3}}-\frac{\lambda^{2} a^{2} d e^{-\lambda a}}{2 K_{3}}-\frac{\lambda^{3} a^{3} d e^{-\lambda a}}{6 K_{3}}-\frac{7 \lambda^{4} a^{4} d e^{-\lambda a}}{720 K_{3}}-\frac{\lambda^{5} a^{5} d e^{-\lambda a}}{1800 K_{3}} \\
& \left.+\frac{\lambda b d e^{-\lambda b}}{K_{3}}+\frac{\lambda^{2} b^{2} d e^{-\lambda b}}{2 K_{3}}+\frac{\lambda^{3} b^{3} d e^{-\lambda b}}{6 K_{3}}+\frac{7 \lambda^{4} b^{4} d e^{-\lambda b}}{720 K_{3}}+\frac{\lambda^{5} b^{5} d e^{-\lambda b}}{1800 K_{3}}\right\} I_{\left(h-\frac{\beta a^{2}}{c}, h-\frac{\beta b^{2}}{d}\right)}(u) \\
& +\left\{\frac{\lambda a e^{-\lambda a}\left(20 a^{2}+5 a^{3} \lambda+a^{4} \lambda^{2}\right)}{60 K_{3}} \frac{\beta}{h-u}+\frac{2 \lambda d \sqrt{d}}{3 K_{3}} \sqrt{\frac{h-u}{\beta}} e^{-\lambda \sqrt{d} \sqrt{\frac{h-u}{\beta}}}\right. \\
& +\frac{5 \lambda^{2} d^{2}}{12 K_{3}} \frac{h-u}{\beta} e^{-\lambda \sqrt{d} \sqrt{\frac{h-u}{\beta}}}+\frac{3 \lambda^{3} d^{2} \sqrt{d}}{20 K_{3}} \sqrt{\frac{h-u}{\beta}} \frac{h-u}{\beta} e^{-\lambda \sqrt{d} \sqrt{\frac{h-u}{\beta}}} \\
& +\frac{7 \lambda^{4} d^{3}}{720 K_{3}}\left(\frac{h-u}{\beta}\right)^{2} e^{-\lambda \sqrt{d} \sqrt{\frac{h-u}{\beta}}}+\frac{\lambda^{5} d^{3} \sqrt{d}}{1800 K_{3}} \sqrt{\frac{h-u}{\beta}}\left(\frac{h-u}{\beta}\right)^{2} e^{-\lambda \sqrt{d} \sqrt{\frac{h-u}{\beta}}} \\
& \left.-\frac{\lambda a d e^{-\lambda a}}{K_{3}}-\frac{\lambda^{2} a^{2} d e^{-\lambda a}}{2 K_{3}}-\frac{\lambda^{3} a^{3} d e^{-\lambda a}}{6 K_{3}}-\frac{7 \lambda^{4} a^{4} d e^{-\lambda a}}{720 K_{3}}-\frac{\lambda^{5} a^{5} d e^{-\lambda a}}{1800 K_{3}}\right\} I_{\left(h-\frac{\beta b^{2}}{d}, h-\frac{\beta a^{2}}{d}\right)}(u)
\end{aligned}
$$

- CASE II $\left(\frac{a^{2}}{c}<\frac{b^{2}}{d}\right)$

$f_{u}(u)=\left\{\frac{\lambda b e^{-\lambda b}\left(20 b^{2}+5 b^{3} \lambda+b^{4} \lambda^{2}\right)}{60 K_{3}} \frac{\beta}{g-u}+\frac{2 \lambda c \sqrt{c}}{3 K_{3}} \sqrt{\frac{g-u}{\beta}} e^{-\lambda \sqrt{c} \sqrt{\frac{g-u}{\beta}}}\right.$$$
+\frac{5 \lambda a^{2} c^{2}}{12 K_{3}} \frac{g-u}{\beta} e^{-\lambda \sqrt{c} \sqrt{\frac{g-u}{\beta}}}+\frac{3 \lambda a^{3} c^{2} \sqrt{c}}{20 K_{3}} \sqrt{\frac{g-u}{\beta} \frac{g-u}{\beta}} e^{-\lambda \sqrt{c} \sqrt{\frac{g-u}{\beta}}}
$$ 


$$
\begin{aligned}
& +\frac{7 \lambda a^{4} c^{2}}{720 K_{3}}\left(\frac{g-u}{\beta}\right)^{2} e^{-\lambda \sqrt{c} \sqrt{\frac{g-u}{\beta}}}+\frac{\lambda a^{5} c^{3} \sqrt{c}}{1800 K_{3}} \sqrt{\frac{g-u}{\beta}}\left(\frac{g-u}{\beta}\right)^{2} e^{-\lambda \sqrt{c} \sqrt{\frac{g-u}{\beta}}} \\
& \left.-\frac{\lambda b c e^{-\lambda b}}{K_{3}}-\frac{\lambda^{2} b^{2} c e^{-\lambda b}}{2 K_{3}}-\frac{\lambda^{3} b^{3} c e^{-\lambda b}}{6 K_{3}}-\frac{7 \lambda^{4} b^{4} c e^{-\lambda b}}{720 K_{3}}-\frac{\lambda^{5} b^{5} c e^{-\lambda b}}{1800 K_{3}}\right\} I_{\left(g-\frac{\beta b^{2}}{c}, g-\frac{\beta b^{2}}{d}\right)}(u) \\
& +\left\{\frac{2 \lambda c \sqrt{c}}{3 K_{3}} \sqrt{\frac{g-u}{\beta}} e^{-\lambda \sqrt{c} \sqrt{\frac{g-u}{\beta}}}+\frac{5 \lambda^{2} c^{2}}{12 K_{3}} \frac{g-u}{\beta} e^{-\lambda \sqrt{c} \sqrt{\frac{g-u}{\beta}}}\right. \\
& +\frac{3 \lambda^{3} c^{2} \sqrt{\mathrm{c}}}{20 K_{3}} \sqrt{\frac{g-u}{\beta} \frac{g-u}{\beta}} e^{-\lambda \sqrt{\mathrm{c}} \sqrt{\frac{g-u}{\beta}}}+\frac{7 \lambda^{4} c^{3}}{720 K_{3}}\left(\frac{g-u}{\beta}\right)^{2} e^{-\lambda \sqrt{c} \sqrt{\frac{g-u}{\beta}}} \\
& +\frac{\lambda^{5} c^{3} \sqrt{c}}{1800 K_{3}} \sqrt{\frac{g-u}{\beta}}\left(\frac{q-u}{\beta}\right)^{2} e^{-\lambda \sqrt{c} \sqrt{\frac{g-u}{\beta}}}-\frac{2 \lambda d \sqrt{d}}{3 K_{3}} \sqrt{\frac{g-u}{\beta}} e^{-\lambda \sqrt{d} \sqrt{\frac{g-u}{\beta}}} \\
& -\frac{5 \lambda^{2} d^{2}}{12 K_{3}} \frac{g-u}{\beta} e^{-\lambda \sqrt{d} \sqrt{\frac{g-u}{\beta}}}-\frac{3 \lambda^{3} d^{2} \sqrt{d}}{20 K_{3}} \sqrt{\frac{q-u}{\beta}} \frac{g-u}{\beta} e^{-\lambda \sqrt{d} \sqrt{\frac{g-u}{\beta}}} \\
& -\frac{7 \lambda^{4} d^{3}}{720 K_{3}}\left(\frac{g-u}{\beta}\right)^{2} e^{-\lambda \sqrt{d} \sqrt{\frac{g-u}{\beta}}}-\frac{\lambda^{5} d^{3} \sqrt{d}}{1800 K_{3}} \sqrt{\frac{g-u}{\beta}}\left(\frac{q-u}{\beta}\right)^{2} e^{-\lambda \sqrt{d} \sqrt{\frac{g-u}{\beta}}} \\
& -\frac{\lambda b c e^{-\lambda b}}{K_{3}}-\frac{\lambda^{2} b^{2} c e^{-\lambda b}}{2 K_{3}}-\frac{\lambda^{3} b^{3} c e^{-\lambda b}}{6 K_{3}}-\frac{7 \lambda^{4} b^{4} c e^{-\lambda b}}{720 K_{3}}-\frac{\lambda^{5} b^{5} c e^{-\lambda b}}{1800 K_{3}} \\
& +\frac{\lambda b d e^{-\lambda b}}{K_{3}}+\frac{\lambda^{2} b^{2} d e^{-\lambda b}}{2 K_{3}}+\frac{\lambda^{3} b^{3} d e^{-\lambda b}}{6 K_{3}}+\frac{7 \lambda^{4} b^{4} d e^{-\lambda b}}{720 K_{3}}+\frac{\lambda^{5} b^{5} d e^{-\lambda b}}{1800 K_{3}} I_{\left(g-\frac{\rho b^{2}}{d}, g-\frac{\theta a^{2}}{c}\right)}(u) \\
& +\left\{-\frac{\lambda a e^{-\lambda a}\left(20 a^{2}+5 a^{3} \lambda+a^{4} \lambda^{2}\right)}{60 K_{3}} \frac{\beta}{g-u}-\frac{2 \lambda d \sqrt{d}}{3 K_{3}} \sqrt{\frac{g-u}{\beta}} e^{-\lambda \sqrt{d} \sqrt{\frac{g-u}{\beta}}}\right. \\
& -\frac{5 \lambda^{2} d^{2}}{12 K_{3}} \frac{q-u}{\beta} e^{-\lambda \sqrt{d} \sqrt{\frac{q-u}{\beta}}}-\frac{3 \lambda^{3} d^{2} \sqrt{d}}{20 K_{3}} \sqrt{\frac{q-u}{\beta}} \frac{q-u}{\beta} e^{-\lambda \sqrt{d} \sqrt{\frac{q-u}{\beta}}} \\
& -\frac{7 \lambda^{4} d^{3}}{720 K_{3}}\left(\frac{g-u}{\beta}\right)^{2} e^{-\lambda \sqrt{d} \sqrt{\frac{\rho-u}{\beta}}}-\frac{\lambda^{5} d^{3} \sqrt{d}}{1800 K_{3}} \sqrt{\frac{g-u}{\beta}\left(\frac{g-u}{\beta}\right)^{2}} e^{-\lambda \sqrt{d} \sqrt{\frac{2-u}{\beta}}} \\
& -\frac{\lambda b c e^{-\lambda b}}{K_{3}}-\frac{\lambda^{2} b^{2} c e^{-\lambda b}}{2 K_{3}}-\frac{\lambda^{3} b^{3} c e^{-\lambda b}}{6 K_{3}}-\frac{7 \lambda^{4} b^{4} c e^{-\lambda b}}{720 K_{3}}-\frac{\lambda^{5} b^{5} c e^{-\lambda b}}{1800 K_{3}} \\
& +\frac{\lambda a c e^{-\lambda a}}{K_{3}}+\frac{\lambda^{2} a^{2} c e^{-\lambda a}}{2 K_{3}}+\frac{\lambda^{3} a^{3} c e^{-\lambda a}}{6 K_{3}}+\frac{7 \lambda^{4} a^{4} c e^{-\lambda a}}{720 K_{3}}+\frac{\lambda^{5} a^{5} c e^{-\lambda a}}{1800 K_{3}} \\
& \left.+\frac{\lambda b d e^{-\lambda b}}{K_{3}}+\frac{\lambda^{2} b^{2} d e^{-\lambda b}}{2 K_{3}}+\frac{\lambda^{3} b^{3} d e^{-\lambda b}}{6 K_{3}}+\frac{7 \lambda^{4} b^{4} d e^{-\lambda b}}{720 K_{3}}+\frac{\lambda^{5} b^{5} d e^{-\lambda b}}{1800 K_{3}}\right\} I_{\left(g-\frac{\beta a^{2}}{c}, g-\frac{\beta a^{2}}{d}\right)}(u) \\
& +\left\{\frac { 1 } { ( e ^ { - \lambda a } - e ^ { - \lambda b } ) ( h - g ) } \left(\lambda b e^{-\lambda b}-\lambda a e^{-\lambda a}+\frac{\lambda^{2} b^{2} e^{-\lambda b}}{2}-\frac{\lambda^{2} a^{2} e^{-\lambda a}}{2}+\frac{\lambda^{3} b^{3} e^{-\lambda b}}{6}-\frac{\lambda^{3} a^{3} e^{-\lambda a}}{6}\right.\right. \\
& \left.\left.+\frac{\lambda^{4} b^{4} e^{-\lambda b}}{720}-\frac{\lambda^{4} a^{4} e^{-\lambda a}}{720}+\frac{\lambda^{5} b^{5} e^{-\lambda b}}{1800}-\frac{\lambda^{5} a^{5} e^{-\lambda a}}{1800}\right)\right\} I_{\left(g-\frac{\beta a^{2}}{d}, h-\frac{\beta b^{2}}{c}\right)}(u) \\
& +\left\{-\frac{\lambda b e^{-\lambda b}\left(20 b^{2}+5 b^{3} \lambda+b^{4} \lambda^{2}\right)}{60 K_{3}} \frac{\beta}{h-u}-\frac{2 \lambda c \sqrt{c}}{3 K_{3}} \sqrt{\frac{h-u}{\beta}} e^{-\lambda \sqrt{c} \sqrt{\frac{h-u}{\beta}}}\right. \\
& -\frac{5 \lambda^{2} c^{2}}{12 K_{3}} \frac{h-u}{\beta} e^{-\lambda \sqrt{c} \sqrt{\frac{h-u}{\beta}}}-\frac{3 \lambda^{3} d^{2} \sqrt{c}}{20 K_{3}} \sqrt{\frac{h-u}{\beta} \frac{h-u}{\beta}} e^{-\lambda \sqrt{c} \sqrt{\frac{h-u}{\beta}}} \\
& -\frac{7 \lambda^{4} c^{3}}{720 K_{3}}\left(\frac{h-u}{\beta}\right)^{2} e^{-\lambda \sqrt{c} \sqrt{\frac{h-u}{\beta}}}-\frac{\lambda^{5} c^{3} \sqrt{c}}{1800 K_{3}} \sqrt{\frac{h-u}{\beta}}\left(\frac{h-u}{\beta}\right)^{2} e^{-\lambda \sqrt{c} \sqrt{\frac{h-u}{\beta}}}
\end{aligned}
$$




$$
\begin{aligned}
& -\frac{\lambda a d e^{-\lambda a}}{K_{3}}-\frac{\lambda^{2} a^{2} d e^{-\lambda a}}{2 K_{3}}-\frac{\lambda^{3} a^{3} d e^{-\lambda a}}{6 K_{3}}-\frac{7 \lambda^{4} a^{4} d e^{-\lambda a}}{720 K_{3}}-\frac{\lambda^{5} a^{5} d e^{-\lambda a}}{1800 K_{3}} \\
& +\frac{\lambda a c e^{-\lambda a}}{K_{3}}+\frac{\lambda^{2} a^{2} c e^{-\lambda a}}{2 K_{3}}+\frac{\lambda^{3} a^{3} c e^{-\lambda a}}{6 K_{3}}+\frac{7 \lambda^{4} a^{4} c e^{-\lambda a}}{720 K_{3}}+\frac{\lambda^{5} a^{5} c e^{-\lambda a}}{1800 K_{3}} \\
& \left.+\frac{\lambda b d e^{-\lambda b}}{K_{3}}+\frac{\lambda^{2} b^{2} d e^{-\lambda b}}{2 K_{3}}+\frac{\lambda^{3} b^{3} d e^{-\lambda b}}{6 K_{3}}+\frac{7 \lambda^{4} b^{4} d e^{-\lambda b}}{720 K_{3}}+\frac{\lambda^{5} b^{5} d e^{-\lambda b}}{1800 K_{3}}\right\} I_{\left(h-\frac{\beta b^{2}}{c}, h-\frac{\beta b^{2}}{d}\right)}(u) \\
& +\left\{\frac{2 \lambda d \sqrt{d}}{3 K_{3}} \sqrt{\frac{h-u}{\beta}} e^{-\lambda \sqrt{d} \sqrt{\frac{h-u}{\beta}}}+\frac{5 \lambda^{2} d^{2}}{12 K_{3}} \frac{h-u}{\beta} e^{-\lambda \sqrt{d} \sqrt{\frac{h-u}{\beta}}}\right. \\
& +\frac{3 \lambda^{3} d^{2} \sqrt{d}}{20 K_{3}} \sqrt{\frac{h-u}{\beta}} \frac{h-u}{\beta} e^{-\lambda \sqrt{d} \sqrt{\frac{h-u}{\beta}}}+\frac{7 \lambda^{4} d^{3}}{720 K_{3}}\left(\frac{h-u}{\beta}\right)^{2} e^{-\lambda \sqrt{d} \sqrt{\frac{h-u}{\beta}}} \\
& +\frac{\lambda^{5} d^{3} \sqrt{d}}{1800 K_{3}} \sqrt{\frac{h-u}{\beta}}\left(\frac{h-u}{\beta}\right)^{2} e^{-\lambda \sqrt{d} \sqrt{\frac{h-u}{\beta}}}-\frac{2 \sqrt{c}}{3 K_{3}} \sqrt{\frac{h-u}{\beta}} e^{-\lambda \sqrt{c} \sqrt{\frac{h-u}{\beta}}} \\
& -\frac{5 \lambda^{2} c^{2}}{12 K_{3}} \frac{h-u}{\beta} e^{-\lambda \sqrt{c} \sqrt{\frac{h-u}{\beta}}}-\frac{3 \lambda^{3} d^{2} \sqrt{c}}{20 K_{3}} \sqrt{\frac{h-u}{\beta}} \frac{h-u}{\beta} e^{-\lambda \sqrt{c} \sqrt{\frac{h-u}{\beta}}} \\
& -\frac{7 \lambda^{4} c^{3}}{720 K_{3}}\left(\frac{h-u}{\beta}\right)^{2} e^{-\lambda \sqrt{c} \sqrt{\frac{h-u}{\beta}}}-\frac{\lambda^{5} c^{3} \sqrt{c}}{1800 K_{3}} \sqrt{\frac{h-u}{\beta}}\left(\frac{h-u}{\beta}\right)^{2} e^{-\lambda \sqrt{c} \sqrt{\frac{h-u}{\beta}}} \\
& -\frac{\lambda a d e^{-\lambda a}}{K_{3}}-\frac{\lambda^{2} a^{2} d e^{-\lambda a}}{2 K_{3}}-\frac{\lambda^{3} a^{3} d e^{-\lambda a}}{6 K_{3}}-\frac{7 \lambda^{4} a^{4} d e^{-\lambda a}}{720 K_{3}}-\frac{\lambda^{5} a^{5} d e^{-\lambda a}}{1800 K_{3}} \\
& \left.+\frac{\lambda a c e^{-\lambda a}}{K_{3}}+\frac{\lambda^{2} a^{2} c e^{-\lambda a}}{2 K_{3}}+\frac{\lambda^{3} a^{3} c e^{-\lambda a}}{6 K_{3}}+\frac{7 \lambda^{4} a^{4} c e^{-\lambda a}}{720 K_{3}}+\frac{\lambda^{5} a^{5} c e^{-\lambda a}}{1800 K_{3}}\right\} I_{\left(h-\frac{\beta b^{2}}{d}, h-\frac{\beta a^{2}}{c}\right)}(u) \\
& +\left\{\frac{\lambda a c e^{-\lambda a}\left(20 a^{2}+5 a^{3} \lambda+a^{4} \lambda^{2}\right)}{60 K_{3}} \frac{\beta}{h-u}+\frac{2 \lambda d \sqrt{d}}{3 K_{3}} \sqrt{\frac{h-u}{\beta}} e^{-\lambda \sqrt{d} \sqrt{\frac{h-u}{\beta}}}\right. \\
& +\frac{5 \lambda^{2} d^{2}}{12 K_{3}} \frac{h-u}{\beta} e^{-\lambda \sqrt{d} \sqrt{\frac{h-u}{\beta}}}+\frac{3 \lambda^{3} d^{2} \sqrt{d}}{20 K_{3}} \sqrt{\frac{h-u}{\beta}} \frac{h-u}{\beta} e^{-\lambda \sqrt{d} \sqrt{\frac{h-u}{\beta}}} \\
& +\frac{7 \lambda^{4} d^{3}}{720 K_{3}}\left(\frac{h-u}{\beta}\right)^{2} e^{-\lambda \sqrt{d} \sqrt{\frac{h-u}{\beta}}}+\frac{\lambda^{5} d^{3} \sqrt{d}}{1800 K_{3}} \sqrt{\frac{h-u}{\beta}}\left(\frac{h-u}{\beta}\right)^{2} e^{-\lambda \sqrt{d} \sqrt{\frac{h-u}{\beta}}} \\
& \left.-\frac{\lambda a d e^{-\lambda a}}{K_{3}}-\frac{\lambda^{2} a^{2} d e^{-\lambda a}}{2 K_{3}}-\frac{\lambda^{3} a^{3} d e^{-\lambda a}}{6 K_{3}}-\frac{7 \lambda^{4} a^{4} d e^{-\lambda a}}{720 K_{3}}-\frac{\lambda^{5} a^{5} d e^{-\lambda a}}{1800 K_{3}}\right\} I_{\left(h-\frac{\beta a^{2}}{c}, h-\frac{\beta a^{2}}{d}\right)}(u)
\end{aligned}
$$

All $f_{U}(u)$ derived so far have the following properties:

- $f_{U}(u) \geq 0$.

- Compute $\int_{-\infty}^{+\infty} f_{U}(u) d u=1$.

- $f_{U}(u)$ is piecewise continuous.

- For any interval $(\mathrm{p}, \mathrm{q}), \operatorname{Pr}(p \leq u \leq q)=\int_{p}^{q} f_{U}(u) d u$.

\subsection{Analysis of probability density functions in multi-pass cases}

Each probability density function has a similar shape with others, but has a different slope. The probability of a surface roughness for the multi-pass cases is determined by the following procedures: 


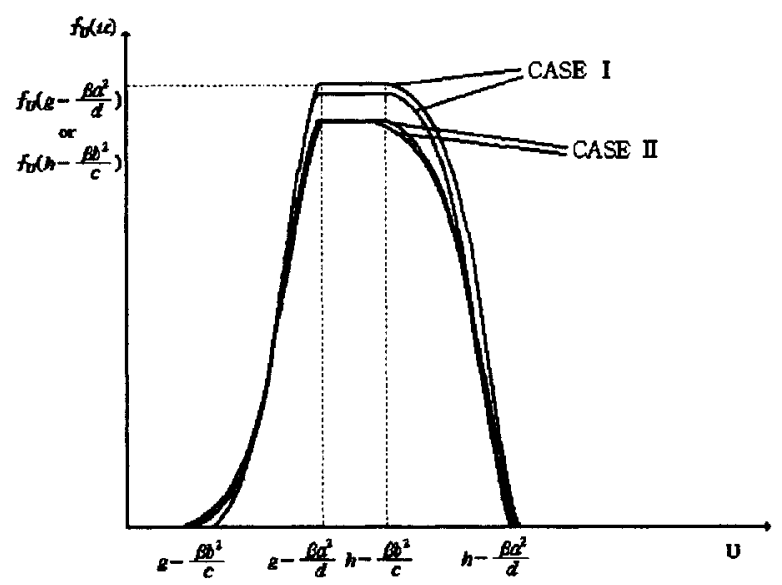

Figure 2: the shape of $f_{U}(u)$

- Define $V_{w}$ and $V_{s}$

- Define $a, b, c, d, h, g$, and $\lambda$

- Specify a surface roughness to be achieved: $p \leq u \leq q$

- Compute $\int_{p}^{q} f_{U}(u) d u$

The detailed results can be summarized for multi-pass cases as follows:

1. If the distribution of $\{X \mid 0<a \leq x \leq b\},\{Y \mid 0<c \leq y \leq d\}$, and $\{T \mid 0<g \leq t \leq h\}$ is given, respectively, all the p.d.f $f_{U}(u)$ are similar shapes having a maximum probability value between $g-\frac{\beta a^{2}}{d}$ and $h-\frac{\beta b^{2}}{c}$ of U. Especially, the probability is constant in the middle stage of a curve when the roughness goes higher. This result can lead to set higher roughness values so that the grinding cost can be reduced.

2. As a reversed situation of 1 ., if $\operatorname{Pr}(p \leq u \leq q)$ is given, the corresponding surface roughness can be estimated in each case. Since the p.d.f $f_{U}(u)$ is a kind of parabolic curves, multiple roughness values are possible with an equal probability. The machining cost would be the most critical factor to select the appropriate surface roughness.

3. If a desired surface roughness along with the probability is given, some critical machining parameters such as $V_{s}$ and $V_{w}$ can be determined. Also, the 
number of passes required to accomplish a given roughness can be derived as follows:

$$
n<\frac{8 c g V_{w}^{2}}{b^{2} V_{w}^{2}}
$$

\section{Measuring Distance and Wheel Radius}

Both the distance between neighboring active grains and the wheel radius of a grinding wheel are the most critical characteristics used for estimating surface roughness in this study. The standard marking system[4] for abrasive wheels has either six or eight parameters dependent on wheel materials, for example, aluminum oxide and silicon carbide or diamond and CBN. Two parameters such as the abrasive grain size and concentration number(or structure) are directly related to this study. The grain size can be obtained by $d_{g}(\mathrm{~mm})=68 M^{-1.4}$ where $M$ is a value of the second parameter in the standard marking system. For the distance between adjacent active grains, the volumetric concentration of abrasive grain in the wheel can be determined first by $D_{g}(\%)=2(32-S)$ where $\mathrm{S}$ is a value of the fourth parameter(concentration number) in the marking system. Then, the distance between the active grains can be found by Equation (2).

$\frac{\pi d_{g}}{\frac{\pi d_{g} D_{g}}{d_{g}}}-d_{g}=\frac{d_{g}}{D_{o}}-d_{g}$

where $d_{g}$ is a wheel diameter.

\section{Conclusion}

The major focus of this study is the probabilistic estimation of surface roughness in the grinding operations when the characteristics of a grinding wheel are specified. The characteristics of the wheel are simplified to two factors: the distance between neighboring active grains and the wheel radius. Although the two characteristics are represented by statistical distributions, other important features of grinding such as vibration, thermal effect, friction, wheel dressing, etc. can be added to the current model for developing more realistic models. The authors have started working on this extension. Either mathematical or probabilistic representations is helpful to develop better grinding conditions, to forecast surface roughness, to minimize manufacturing cost and to be competitive in product quality.

\section{Acknowledgement}

The authors thanks to the Korea Science and Engineering Foundation for its financial support to this research. Research number is 961-1012-089-1 


\section{Biography}

Hoo-Gon Choi is a professor in the Department of Industrial Engineering at Sung Kyun Kwan University in S. Korea. He received BS and MS degrees from Seoul National University, and $\mathrm{MS}$ and $\mathrm{PhD}$ degrees from Iowa State University. His research interests and publications are in CAPP and CIMS. He is a senior member of AIIE and SME.

Myung-Koo Yeo is a doctoral student in the Department of Industrial Engineering at Sung Kyun Kwan University in S. Korea. He received his BS and MS degrees from Sung Kyun Kwan University. His research interests are in Machining and CAPP.

Jae-Yoon Kim is a graduate student in the Department of Industrial Engineering at Sung Kyun Kwan University in S. Korea. He received his BS degree from Sung Kyun Kwan University. His research interests are in Machining and CAPP.

\section{References}

[1] Boothroyd, G., (1975)Fundamentals of Metal Machining and Machine Tools, Scripta Book Company, Chap. 1, 5, 10.

[2] Kannappan, S., and Malkin, S., (1972) "Effects of Grain Size and Operating Parameters on the Mechanics of Grinding", Journal of Engineering for Industry, pp. 833-842.

[3] Law, S.S., Wu, S.M., and Joglekar, A.M., (1973) "On Building Models for the Grinding Process", Journal of Engineering for Industry, pp. 983-991.

[4] Malkin, S., (1989) GRINDING TECHNOLOGY - Theory and Applications of Machining with Abrasives. Ellis Horwood Ltd, Chap. 1, 2, 3, 4, 7.

[5] Peters, J., Inasaki, I., Paul, T., and Tonshoff, H.K., ( 1992 ) "Modeling and Simulation of Grinding Processes", Annals of the CIRP, VOL. 41, NO. 2, pp. $677-688$.

[6] Xiao, G., Malkin, S., and Danai, K., ( 1992 ) "Intelligent Control of Cylindrical Plunge Grinding",Proc. of 1992 American Control Conference, pp. 391-398. 